\title{
Origin of the geometric tachyon
}

\author{
Ashok Das ${ }^{a, c}$, Sudhakar Panda ${ }^{b}$ and Shibaji Roy ${ }^{c *}$ \\ ${ }^{a}$ Department of Physics and Astronomy, University of Rochester, Rochester, NY 14627, USA \\ ${ }^{b}$ Harish-Chandra Research Institute, Chhatnag Road, Jhusi, Allahabad 211019, India and \\ ${ }^{c}$ Saha Institute of Nuclear Physics, 1/AF Bidhannagar, Calcutta-700 064, India
}

\begin{abstract}
The motion of a D $p$-brane in the background of a stack of coincident NS5-branes is analyzed as the motion of a relativistic point particle in the transverse space of the five-branes. In this system, the particle experiences a proper acceleration orthogonal to its proper velocity due to the background dilaton field which changes the dynamics from that of a simple geodesic motion. In particular, we show that in the vicinity of the five-branes, it is this acceleration which is responsible for modifying the motion of the radial mode to that of an inverted simple harmonic oscillator leading to the tachyonic instability.
\end{abstract}

PACS numbers: 11.25.-w, 11.25.Uv, 04.65.+e

In string theory, it is well-known that soliton solutions like the NS5-brane and Dp-brane are contained in the string spectrum. While the former is supersymmetric and stable, the latter can be either supersymmetric and stable (BPS D p-brane) or non-supersymmetric and unstable (non-BPS D $p$-brane). A pair of $\mathrm{D}-\overline{\mathrm{D}}$ branes is non-supersymmetric even if each is supersymmetric individually. Such non-supersymmetric systems are unstable because the lowest lying state of the open string, either (both ends) ending on a single non-BPS D-brane or stretched between the brane and the antibrane, is a tachyon. The condensation of the tachyon can lead to a stable brane configuration or the total annihilation of the brane. A nice review of this phenomenon can be found in [1]. The dynamics of tachyon condensation has led to many interesting time dependent phenomena including the decay of a non-BPS brane into a strange "tachyon matter" state whose equation of state is that of a pressureless fluid [2]. An effective action of the Dirac-BornInfeld (DBI) type for the tachyon [3, 4, 5, 6] has been very useful in understanding such processes.

A completely different dynamics, namely, that of a BPS D $p$-brane propagating in the background of a stack of coincident NS5-branes, has been studied recently using the effective DBI action [7]. It has been observed there that when the $\mathrm{D} p$-brane comes close to the NS5branes, the dynamics of the $\mathrm{D} p$-brane can be mapped to that of the open string tachyon condensation, where the radial mode on the $\mathrm{D} p$-brane plays the role of the tachyon. Moreover, its equation of state approaches that of a pressureless fluid with the pressure falling off exponentially at late times. These properties along with the fact that a parallel D $p$-NS5 brane system is a nonsupersymmetric system, has led to the nomenclature "geometric tachyon" for the radial mode on the $\mathrm{D} p$-brane. The tachyonic instability has been made more precise [8] by compactifying one of the transverse directions of

*e-mails: das@pas.rochester.edu,_ panda@mri.ernet.in, shibaji.roy@saha.ac.in the NS5-branes on a circle and placing the $\mathrm{D} p$-brane, as a point on this circle diametrically opposite to the fivebranes. In such a case it has been observed that the potential energy density of the $\mathrm{D} p$-brane at this point has a saddle point. As a result, this point corresponds to an unstable equilibrium and the $\mathrm{D} p$-brane develops a tachyonic mode associated with translations along the circle. Various other aspects of this system have also been investigated in 9 , 10, 11, 12, 13, 14, 15]. For example, it has been noted recently that under certain conditions a geometric tachyon in one system gets mapped to the universal open string tachyon in another system and viceversa [16].

In this letter we look for a better understanding of the origin of the tachyonic instability when the motion is in the uncompactified transverse space. Our interest is in the dynamics of a BPS D $p$-brane in type II string theory in the presence of $N$ coincident NS5-branes and we formulate this as the motion of a relativistic point particle in the background of fields generated by the NS5 branes. We take the five-branes to be stretched in the directions $\left(x^{1}, x^{2}, \ldots, x^{5}\right)$ and their world-volume directions are denoted by $x^{\bar{\mu}}, \bar{\mu}=0,1, \ldots, 5$. Similarly the transverse directions are labeled by $x^{m}, m=6,7,8,9$. The $\mathrm{D} p$-brane is taken to be parallel to the coincident five-branes, i.e., it is extended in the directions $x^{\mu}, \mu=0,1, \ldots, p$ with $p \leq 5$. Hence the $\mathrm{D} p$-brane is point-like in the directions $x^{m}$. When the $\mathrm{D} p$-brane is placed at a large distance $r=\left(x^{m} x^{m}\right)^{1 / 2}$, from the stack of coincident NS5-branes, it experiences an attractive force due to both gravitational and dilatonic interactions. Since the mass $\left(\sim 1 / g_{s}^{2}\right.$ where $g_{s}$ denotes the string coupling constant) of the NS5-branes is much larger than the mass $\left(\sim 1 / g_{s}\right)$ of the $\mathrm{D} p$-brane at weak string coupling, the $\mathrm{D} p$-brane will move towards the NS5-branes.

The background fields for the five-branes are obtained from the supergravity solution as $\left(\eta_{\bar{\mu} \bar{\nu}}=(-,+, \cdots,+)\right)$

$$
\begin{aligned}
d s^{2} & =-d \tau^{2}=\eta_{\bar{\mu} \bar{\nu}} d x^{\bar{\mu}} d x^{\bar{\nu}}+G_{m n} d x^{m} d x^{n}, \\
G_{m n} & =H(r) \delta_{m n}, \\
e^{2\left(\phi-\phi_{0}\right)} & =H(r)=1+\frac{N \ell_{s}^{2}}{r^{2}},
\end{aligned}
$$




$$
H_{m n p}=-\epsilon_{m n p}^{q} \partial_{q} \phi
$$

where $H(r)$ is the harmonic function describing the $N$ coincident five-branes (with $\ell_{s}$ denoting the fundamental string length). Here $\phi$ denotes the dilaton field, $e^{\phi_{0}}=g_{s}$ is the string coupling constant and $H_{m n p}$ is the fieldstrength of the NS $B$-field.

We label the world-volume coordinates of the $\mathrm{D} p$-brane by $\xi^{\mu}, \mu=0,1, \ldots, p$ and identify $\xi^{\mu}=x^{\mu}$ by making use of the reparametrization invariance on the world-volume. Since, we are considering the motion of the $\mathrm{D} p$-brane only in the transverse space of the NS5-branes, the position of the $\mathrm{D} p$-brane in this space, $\left(x^{6}, x^{7}, x^{8}, x^{9}\right)$, gives rise to scalar fields, $X^{m}\left(\xi^{\mu}\right), m=6, \ldots, 9$ on the world-volume of the $\mathrm{D} p$-brane. The dynamics of these scalar fields is governed by the DBI action

$$
S_{p}=-\tau_{p} \int d^{p+1} \xi e^{-\left(\phi-\phi_{0}\right)} \sqrt{-\operatorname{det}\left(G_{\mu \nu}+B_{\mu \nu}\right)}
$$

where $\tau_{p}$ is the tension of the $\mathrm{D} p$-brane. $G_{\mu \nu}$ and $B_{\mu \nu}$ are the induced metric and the $B$-field on the world-volume, given by $\left(X^{\bar{\mu}}=x^{\bar{\mu}}\right)$

$$
\begin{aligned}
G_{\mu \nu} & =\frac{\partial X^{A}}{\partial \xi^{\mu}} \frac{\partial X^{B}}{\partial \xi^{\nu}} G_{A B}(X) \\
B_{\mu \nu} & =\frac{\partial X^{A}}{\partial \xi^{\mu}} \frac{\partial X^{B}}{\partial \xi^{\nu}} B_{A B}(X) .
\end{aligned}
$$

Here the indices $A, B=0,1, \ldots, 9$ and $G_{A B}$ and $B_{A B}$ are the metric and the $B$-field in ten dimensions, given in (1). We are interested in the case when the fields representing the position of the $\mathrm{D} p$-brane $X^{m}, m=6, \ldots, 9$, depend only on time, $X^{m}=X^{m}(t)$. In this case, the action (2) simplifies considerably and takes the form

$$
S_{p}=-\tau_{p} V_{p} \int d t e^{-\left(\phi-\phi_{0}\right)} \sqrt{1-G_{m n} \dot{X}^{m} \dot{X}^{n}}
$$

where $V_{p}$ is the volume of the $p$-dimensional space in which the $\mathrm{D} p$-brane is stretched out and an 'overdot' represents a derivative with respect to $t$. The dilaton field and the metric $G_{m n}$ are related to the harmonic function as noted in (11).

We rewrite the above action in a suggestive form as,

$$
\begin{aligned}
S_{p} & =-\tau_{p} V_{p} \int d t e^{-\bar{\phi}} \sqrt{-G_{\bar{m} \bar{n}} \dot{X}^{\bar{m}} \dot{X}^{\bar{n}}} \\
& =-\tau_{p} V_{p} \int d \tau e^{-\bar{\phi}} \sqrt{-G_{\bar{m} \bar{n}} \frac{d X^{\bar{m}}}{d \tau} \frac{d X^{\bar{n}}}{d \tau}}
\end{aligned}
$$

where $\bar{\phi}=\phi-\phi_{0}, G_{\bar{m} \bar{n}}=\left(-1, G_{m n}\right)$ with $\bar{m}$ and $\bar{n}$ taking values $\bar{m}=(0, m)=0,6,7,8,9 ; X^{0}=t$ and $\tau$ is the proper time. The action (5) can be thought of as describing the dynamics of a relativistic point particle in gravitational as well as dilatonic backgrounds. Note that $\bar{\phi}$ does not depend on $X^{0}$ and, therefore, on time explicitly. However, since it depends upon $X^{m}$, which is a function of time, it has an implicit time dependence. The Lorentz factor follows from (1) to be

$$
\gamma=\frac{d t}{d \tau}=\frac{1}{\sqrt{-G_{\bar{m} \bar{n}} \dot{X}^{\bar{m}} \dot{X}^{\bar{n}}}}
$$

and denoting the proper velocity of the particle as $u^{\bar{m}}=$ $d X^{\bar{m}} / d \tau$, it can be checked easily that $G_{\bar{m} \bar{n}} u^{\bar{m}} u^{\bar{n}}=-1$. The momentum of the particle is obtained from (5) to be $P_{\bar{m}}=\tau_{p} V_{p} e^{-\bar{\phi}} G_{\bar{m} \bar{n}} u^{\bar{n}}$, which leads to $P^{2}=G^{\bar{m} \bar{n}} P_{\bar{m}} P_{\bar{n}}=$ $-\left(\tau_{p} V_{p}\right)^{2} e^{-2 \bar{\phi}}$ and this makes it clear that such a particle is not tachyonic in the conventional sense unless the dilaton field becomes complex.

Therefore, to understand the origin of the tachyonic instability, let us analyze the equations of motion following from (5) which correspond to the motion of a particle in a curved background subject to an acceleration, namely,

$$
\frac{d^{2} X^{\bar{m}}}{d \tau^{2}}+\Gamma_{\bar{n} \bar{p}}^{\bar{m}} \frac{d X^{\bar{n}}}{d \tau} \frac{d X^{\bar{p}}}{d \tau}=\alpha^{\bar{m}}
$$

where $\Gamma_{\bar{n}}^{\bar{m}}$ is the Christoffel symbol constructed from the metric $G_{\bar{m} \bar{n}}$, namely,

$$
\Gamma_{\bar{n} \bar{p}}^{\bar{m}}=\frac{1}{2} G^{\bar{m} \bar{q}}\left(\partial_{\bar{n}} G_{\bar{q} \bar{p}}+\partial_{\bar{p}} G_{\bar{n} \bar{q}}-\partial_{\bar{q}} G_{\bar{n} \bar{p}}\right),
$$

and the proper acceleration $\alpha^{\bar{m}}$ is given by

$$
\alpha^{\bar{m}}=\left(G^{\bar{m} \bar{n}}+\frac{d X^{\bar{m}}}{d \tau} \frac{d X^{\bar{n}}}{d \tau}\right) \partial_{\bar{n}} \bar{\phi} .
$$

Thus we note that the dilaton background is responsible for a proper acceleration leading to a deviation of the trajectory of the particle from its geodesic. It can be checked easily that $G_{\bar{m} \bar{n}} u^{\bar{m}} \alpha^{\bar{n}}=0$ so that the proper acceleration is orthogonal to the proper velocity as would be expected for a relativistic system. This is reminiscent of a Rindler particle executing hyperbolic motion [17] and clarifies the origin of the hyperbolic solution obtained in 7].

We can compute the energy-momentum tensor by using the general formula

$$
T^{\mu \nu}=-\frac{\partial L}{\partial\left(\partial_{\mu} X^{m}\right)} \partial^{\nu} X^{m}+\eta^{\mu \nu} L
$$

where $L$ is the Lagrangian of the action (5) and the nonvanishing components take the explicit forms,

$$
\begin{aligned}
T^{00} & =\tau_{p} V_{p} \gamma e^{-\bar{\phi}} \equiv E \\
T^{i j} & =-\tau_{p} V_{p} \gamma^{-1} e^{-\bar{\phi}} \delta^{i j} \equiv p \delta^{i j},
\end{aligned}
$$

where $\gamma$ is the Lorentz factor defined in (6) and $E, p$ denote the energy and the pressure of the system respectively. From time translation invariance we expect energy to be conserved and similarly rotational invariance in the transverse space leads to the conservation of angular momentum in the system. 
The time component $(\bar{m}=0)$ of the equation of motion (77) yields

$$
\frac{d \gamma}{d t}=\gamma \frac{d \bar{\phi}}{d t}
$$

which we recognize from (11) to lead to conservation of energy. On the other hand, using (12) the dynamical equation (7) for $\bar{m}=m$ takes the form

$$
\ddot{X}^{m}+\Gamma_{\bar{n} \bar{p}}^{m} \dot{X}^{\bar{n}} \dot{X}^{\bar{p}}+G_{\bar{p} \bar{q}} \dot{X}^{\bar{p}} \dot{X}^{\bar{q}} G^{m n} \partial_{n} \bar{\phi}=0 .
$$

We note that for large separations the leading behaviour of this equation is the free particle motion described by $\ddot{X}^{m}=0$. This corresponds to the vanishing of the gravitational force as well as the acceleration $\alpha^{m}$ for large separations in the leading order. We study below the dynamics of the system in the next to leading order.

For this purpose it is simpler to work in the sphericalpolar coordinates. Using the fact that angular momentum is conserved, we can restrict the motion of the particle to a plane with the radial mode $R$ and the angular mode $\Theta$. In this case the line element in (1) takes the form

$$
-d \tau^{2}=-d t^{2}+H d R^{2}+H R^{2} d \Theta^{2},
$$

and correspondingly the Lorentz factor (6) becomes,

$$
\gamma=\frac{1}{\sqrt{1-H \dot{R}^{2}-H R^{2} \dot{\Theta}^{2}}}
$$

The nonvanishing components of $\Gamma$ can be computed from (8) and are given by,

$$
\begin{aligned}
& \Gamma_{R R}^{R}=\partial_{R} \ln \sqrt{H}, \quad \Gamma_{\Theta \Theta}^{R}=-R^{2} \partial_{R} \ln \sqrt{H R^{2}}, \\
& \Gamma_{R \Theta}^{\Theta}=\Gamma_{\Theta R}^{\Theta}=\partial_{R} \ln \sqrt{H R^{2}} .
\end{aligned}
$$

In the spherical coordinates (13) has the form

$$
\begin{aligned}
\ddot{R}-R \dot{\Theta}^{2}+\frac{1}{2 H^{2}} \partial_{R} H\left(2 H \dot{R}^{2}-1\right) & =0, \\
\ddot{\Theta}+\frac{1}{H R^{2}} \partial_{R}\left(H R^{2}\right) \dot{\Theta} \dot{R} & =0 .
\end{aligned}
$$

Since $\Theta$ is an angular coordinate, its conjugate gives the angular momentum of the form

$$
L=\tau_{p} V_{p} \gamma e^{-\bar{\phi}} H R^{2} \dot{\Theta}=E H R^{2} \dot{\Theta} .
$$

Defining the quantity

$$
H R^{2} \dot{\Theta}=\frac{L}{E}=\ell,
$$

we note that the second equation in (17) leads to the conservation condition

$$
\frac{d}{d t}\left(H R^{2} \dot{\Theta}\right)=\frac{d}{d t}\left(\frac{L}{E}\right)=\frac{d \ell}{d t}=0,
$$

namely, the angular momentum associated with the motion of the particle is conserved.

The true dynamics of the system is contained in the $R$-equation in (17). Using (18) as well as the fact that energy in (11) is conserved, we obtain from (15)

$$
\dot{R}^{2}=\frac{1}{H}\left(1-\frac{1}{H}\left(\left(\frac{\tau_{p} V_{p}}{E}\right)^{2}+\frac{\ell^{2}}{R^{2}}\right)\right) \geq 0,
$$

which determines (using the form of $H$ in (10) that for $\frac{\tau_{p} V_{p}}{E} \geq 1$, we must have $\left(N \ell_{s}^{2}-\ell^{2}\right) \geq 0$ and $R^{2} \leq R_{0}^{2}$, while for $\frac{\tau_{p} V_{p}}{E} \leq 1$, we can have either $\left(N \ell_{s}^{2}-\ell^{2}\right) \geq 0$ without any restriction on $R$, or $\left(N \ell_{s}^{2}-\ell^{2}\right) \leq 0$ with $R^{2} \geq R_{0}^{2}$ where $R_{0}^{2}=\left|\frac{N \ell_{s}^{2}-\ell^{2}}{\left(\frac{\tau_{p} V_{p}}{E}\right)^{2}-1}\right|$. Since we are interested in the behavior of the system close to the origin $R \simeq 0$, it is clear that we must have $\left(N \ell_{s}^{2}-\ell^{2}\right) \geq 0$ independent of the value of the ratio $\frac{\tau_{p} V_{p}}{E}$. (Parenthetically, we remark here that the analysis in [7] assumed that $\frac{\tau_{p} V_{p}}{E} \geq 1$, but the difference in whether this ratio is bigger than or smaller than unity simply reflects how far away the D $p$-brane can be from the NS5 branes initially. Since it is natural to assume that the $\mathrm{D} p$-brane starts out infinitely far away, we would assume $\frac{\tau_{p} V_{p}}{E} \leq 1$, although what is really important for the analysis of the behavior near the NS5 branes is that $N \ell_{s}^{2}-\ell^{2} \geq 0$.)

From (16) as well as (9) we find that for large $R$ both the gravitational as well as the dilatonic forces behave as $\frac{1}{R^{3}}$ so that for large $R \gg \sqrt{N} \ell_{s}$ the radial equation in (17) yields

$$
\ddot{R}+\left(N \ell_{s}^{2}-\ell^{2}\right)\left(-\frac{2 N \ell_{s}^{2}}{R_{0}^{2}}+1\right) \frac{1}{R^{3}}=0,
$$

as expected for a particle moving in an attractive $-1 / R^{2}$ potential (up to multiplicative factors) in four spatial dimensions (as long as $\left(N \ell_{s}^{2}-\ell^{2}\right)>0$ ). The dynamics of the $\mathrm{D} p$-brane in the vicinity $\left(R \ll \sqrt{N} \ell_{s}\right)$ of the NS5-branes is better understood in the variable $Z=1 / R$. In this variable the harmonic function becomes $H=1+N \ell_{s}^{2} Z^{2}$ and for $\sqrt{N} \ell_{s} Z \gg 1$, the $R$ equation in (17) takes the form

$$
\ddot{Z}-\frac{1}{\left(N \ell_{s}^{2}\right)^{2}}\left(N \ell_{s}^{2}-\ell^{2}\right) Z=0 .
$$

For $\left(N \ell_{s}^{2}-\ell^{2}\right)>0$ which is the case of interest for us, we recognize (23) to correspond to an inverted simple harmonic oscillator. On the other hand, we note that in the absence of the acceleration the radial equation for small $R$ reduces to $\ddot{Z}=0$. The origin of the tachyonic instability is now clear, namely, it is the acceleration due to the dilatonic background which is the source of the instability. Even though $P^{2}<0$ indicating that the particle is not tachyonic in the conventional sense, the dilatonic force that it experiences in the background of the NS5 branes leads to hyperbolic motion and the tachyonic instability 
in the system. Furthermore, using this hyperbolic motion (solution of (23) ), we can check the known fact that the pressure as given in (11) falls off exponentially at late time. We note here that although (23) exhibits a tachyonic instability, in order to see where the instability occurs we give the complete radial equation below (see the first eq. in (17))

$$
\begin{aligned}
\ddot{R} & =\frac{\left(N \ell_{s}^{2}-\ell^{2}\right) N \ell_{s}^{2}}{\left(R^{2}+N \ell_{s}^{2}\right)^{3}}\left(R+\left(\frac{2}{R_{0}^{2}}-\frac{1}{N \ell_{s}^{2}}\right) R^{3}\right) \\
& =-\frac{d V}{d R}
\end{aligned}
$$

where $V(R)$ is the potential in which the particle moves. From this we find that, for $R_{0}<\sqrt{N} \ell_{s}$ which is the case of our interest, the instability occurs at $R=0$ and the effective mass squared of the particle is

$$
\left.m^{2} \equiv \frac{d^{2} V(R)}{d R^{2}}\right|_{R=0}=-\frac{\left(N \ell_{s}^{2}-\ell^{2}\right)}{\left(N \ell_{s}^{2}\right)^{2}}
$$

However, since the effective string coupling $e^{\bar{\phi}}$ blows up at $R=0$, a full quantum treatment is necessary to identify the true position of instability.

We emphasize that in the absence of the additional force produced by the dilaton, the particle will follow a gravitational geodesic which does not lead to any instability. Of course, this cannot happen within the context of string theory unless we set the dilaton to zero by hand. On the other hand, if we consider the motion of a fundamental string, given by the Nambu-Goto action, in the background of a stack of coincident D5-branes, then the absence of a dilaton prefactor in the Nambu-Goto action may lead one to think that the particle will not experience any acceleration and would follow the gravitational geodesic without any instability. However, this is not true since the background D5-branes can give rise to an induced metric with an overall conformal factor (so that the action takes the form (5) ) and this conformal factor can indeed be a source of proper acceleration leading to a geometric tachyon in the dynamics. In fact, it is known that a parallel but separated F-D5 system is nonsupersymmetric and so we expect a tachyonic instability. The F-string in this case is known to melt into the D5branes to form a non-threshold bound state [18]. The formation of this bound state can be viewed as due to the geometric tachyon condensation. The details of this work will be published in a companion paper [19].

This work was supported in part by US DOE Grant number DE-FG 02-91ER40685. Two of us (S.P. and S.R.) would like to thank the Department of Physics and Astronomy of the University of Rochester, where this work is done, for the warm hospitality.
[1] A. Sen, Int. J. Mod. Phys. A 20, 5513 (2005) arXiv:hep-th/0410103.

[2] A. Sen, JHEP 0207, 065 (2002) arXiv:hep-th/0203265].

[3] A. Sen, JHEP 9910, 008 (1999) arXiv:hep-th/9909062.

[4] M. R. Garousi, Nucl. Phys. B 584, 284 (2000) arXiv:hep-th/0003122.

[5] E. A. Bergshoeff, M. de Roo, T. C. de Wit, E. Eyras and S. Panda, JHEP 0005, 009 (2000) arXiv:hep-th/0003221.

[6] J. Kluson, Phys. Rev. D 62, 126003 (2000) arXiv:hep-th/0004106.

[7] D. Kutasov, arXiv:hep-th/0405058

[8] D. Kutasov, arXiv:hep-th/0408073

[9] J. Kluson, JHEP $\mathbf{0 5 0 3 ,} 044$ arXiv:hep-th/0501010.

[10] B. Chen, M. Li and B. Sun, JHEP 0412, 057 (2004) arXiv:hep-th/0412022.
[11] B. Chen and B. Sun, Phys. Rev. D 72, 046005 (2005) arXiv:hep-th/0501176.

[12] S. Thomas and J. Ward, JHEP 0510, 098 (2005) arXiv:hep-th/0502228.

[13] S. Thomas and J. Ward, Phys. Rev. D 72, 083519 (2005) arXiv:hep-th/0504226.

[14] S. Panda, M. Sami and S. Tsujikawa, Phys. Rev. D 73, 023515 (2006) arXiv:hep-th/0510112.

[15] K. L. Panigrahi and H. Singh, JHEP 0711, 017 (2007) arXiv:0708.1679 [hep-th]].

[16] A. Sen, JHEP 0705, 035 (2007) arXiv:hep-th/0703157.

[17] W. Rindler, Phys. Rev. 119, 2082 (1960); Amer. Jour. of Phys. 34, 1174 (1966).

[18] J. X. Lu and S. Roy, Nucl. Phys. B 560, 181 (1999) arXiv:hep-th/9904129.

[19] A. Das, S. Panda and S. Roy, in preparation. 